\title{
MODELO BAYESIANO DE PRODUCCIÓN EXCEDENTE CON AUTOCORRELACIÓN SERIAL
}

\author{
DANiel R. HernándeZ ${ }^{\dagger}$ y JULIETA S. RodRíGueZ ${ }^{1}$ \\ Instituto Nacional de Investigación y Desarrollo Pesquero (INIDEP), \\ Paseo Victoria Ocampo No 1, Escollera Norte, B7602HSA - Mar del Plata, Argentina \\ ${ }^{1}$ correo electrónico: julieta@inidep.edu.ar
}

\begin{abstract}
RESUMEN. Se presenta un modelo simple de producción excedente al que se denomina Modelo de Producción Excedente con Autocorrelación Serial (MPECAS) debido a que considera como único supuesto que la producción excedente presenta correlación serial y no tiene una relación funcional explícita con la biomasa. Su aplicación se logra solo con un índice de abundancia proporcional a una potencia dada de la abundancia media real del recurso y la serie de capturas anuales correspondientes. La estimación de los parámetros del modelo se plantea en un contexto bayesiano utilizando el algoritmo SIR (Sampling Importance Resampling). Se proponen criterios de riesgo sencillos para estimar la Captura Máxima Biológicamente Aceptable (CMBA) y los riesgos asociados a cada nivel de captura hipotética considerada. Se llevó a cabo un ejercicio de simulación para evaluar la capacidad estadística del MPECAS para reproducir la información proporcionada por un modelo operacional de producción excedente de Schaefer considerado como real. Finalmente, se presenta un ejemplo de aplicación con el recurso corvina rubia (Micropogonias furnieri) y se muestran las CMBA para el 5 y 10\% de riesgo de disminución de biomasa en el año siguiente al de evaluación calculadas con el modelo de Schaefer y el MPECAS.
\end{abstract}

Palabras clave: Producción excedente, autocorrelación serial, evaluación de recursos, estimación bayesiana, Micropogonias furnieri.

\section{BAYESIAN SURPLUS PRODUCTION MODEL WITH SERIAL AUTOCORRELATION}

ABSTRACT. Presentation is made of a simple surplus production model called Surplus Production Model with Serial Autocorrelation (MPECAS in Spanish) since it considers as a unique assumption that the surplus production shows a serial correlation and has no explicit functional relation with biomass. Its application requires only an abundance index proportional to a given power of the actual mean abundance of the resource and the corresponding annual catches series. The estimate of the model parameters is presented within a Bayesian context using the SIR (Sampling Importance Resampling) algorithm. Simple risk criteria are proposed to estimate the Maximum Biologically Acceptable Catch (MBAC) and the risks associated to each hypothetical catch level considered. A simulation exercise was performed to assess the statistical capability of MPECAS to reproduce the information provided by a Schaefer operational surplus production model considered as an actual one. Finally, an application example with the white croaker (Micropogonias furnieri) is presented and the MBAC for 5 and 10\% risk of biomass decline the year following the assessment year calculated with the Schaefer and MPECAS models are shown.

Key words: Surplus production, serial autocorrelation, stock assessment, Bayesian estimate, Micropogonias furnieri.

†El Lic. Daniel R. Hernández falleció el 25 de enero de 2019. 


\section{INTRODUCCIÓN}

Los modelos de producción excedente, como el de Schaefer, Fox y Pella y Tomlinson (Hilborn y Walters 1992) son ampliamente utilizados desde hace décadas en la modelación de poblaciones de peces para una gran variedad de especies en todo el mundo. Si bien este tipo de aproximaciones es de gran importancia porque permite obtener estimaciones de biomasa en especies con datos pobres, requieren de relaciones paramétricas explícitas entre la producción excedente y la biomasa del recurso.

El objetivo de este trabajo es presentar y aplicar con datos reales el denominado Modelo de Producción Excedente con Autocorrelación Serial (MPECAS), basado en el supuesto de que la producción excedente presenta correlación serial y no tiene una relación funcional explícita con la biomasa, requiriendo para su aplicación solo un índice de abundancia proporcional a una potencia dada de la abundancia media real del recurso y las correspondientes capturas anuales. MPECAS es un modelo simple de producción excedente que no requiere de supuestos fuertes para determinar los niveles precautorios de Capturas Máximas Biológicamente Aceptables (CMBA).

El modelo planteado permite efectuar proyecciones de biomasa en un corto-mediano plazo (de 1 a 5 años) y evaluar situaciones futuras probables del recurso ante una secuencia de capturas preestablecidas. Si bien el modelo planteado requiere de la estimación de ciertos parámetros que determinan en parte la evolución de las trayectorias de biomasa, el propósito no es la estimación en sí del vector de parámetros, sino utilizar el modelo para efectuar proyecciones y poder así efectuar análisis de riesgos que permitan la estimación de capturas precautorias según diferentes criterios.

La estimación de los parámetros del modelo se plantea en un contexto bayesiano y se considera el algoritmo SIR (Sampling Importance Resampling) (Bernardo y Smith 2000) como herramienta de cálculo numérico, a los efectos de determinar las distribuciones a posteriori de los mismos.

Se proponen cuatro criterios de riesgo simples. Los cuales permiten determinar CMBA, calculadas en forma tal de mantener los riesgos menores a valores preestablecidos (generalmente inferior al 5 o $10 \%$ ).

Se llevó a cabo un ejercicio de simulación para evaluar la capacidad estadística del MPECAS para reproducir la información proporcionada por un modelo operacional de producción excedente de Schaefer considerado como real.

Se realizó la implementación del MPECAS para el recurso corvina rubia (Micropogonias furnieri), estimándose de modo satisfactorio las CMBA para el 5 y $10 \%$ de riesgo de disminución de biomasa el año siguiente al año de evaluación. La implementación es destacable por respetar la estructura básica de un modelo de producción excedente, prescindiendo de supuestos fuertes y restrictivos, no siempre fácilmente justificables.

\section{MATERIALES Y MÉTODOS}

El modelo de producción excedente lo escribimos en su forma general como:

$B_{t}=B_{t-1}+P_{t-1}-C_{t-1}$

siendo $B_{t}$ y $B_{t+1}$, las biomasas del stock explotable al comienzo de los años $t$ y $t+1$, respectivamente y $P_{t-1}$ y $C_{t-1}$ la producción excedente y la captura total durante el año $t-1$, respectivamente.

Además, consideraremos que la producción excedente evoluciona según el proceso autoregresivo de primer orden AR(1) (Harvey 1981), el cual se puede expresar en la forma:

$P_{t}=\mu(1-\rho)+\rho P_{t-1}+\sigma_{\varepsilon} \varepsilon_{t}$ 
siendo:

$\rho$ : coeficiente de autocorrelación serial de primer orden de la secuencia de producciones excedentes, $|\rho|<1$;

$\sigma_{\varepsilon}:$ desvío estándar del error estructural en (2); $\varepsilon_{t}$ : secuencia de variables aleatorias independientes, teniendo cada una distribución normal con media 0 y desvío estándar 1 .

La condición $|\rho|<1$ determina que el modelo sea estacionario de segundo orden con el valor esperado y la varianza de la producción excedente dados por:

$$
\begin{aligned}
& E\left(P_{t}\right)=\mu \\
& \sigma^{2}\left(P_{t}\right)=\frac{\sigma_{\varepsilon}^{2}}{\left(1-\rho^{2}\right)} .
\end{aligned}
$$

En caso de ser $\rho=1$ el modelo (2) se transforma en un modelo de caminata al azar (random walk) y deja de ser estacionario en la varianza, teniendo la capacidad de variar en forma no acotada (Harvey, 1981).

Además de las ecuaciones (1) y (2), a los efectos de poder efectuar la estimación de parámetros, consideramos la ecuación de observación, con error de observación:

$I_{t}=q \bar{B}_{t}^{\beta} e^{\eta_{t}}$

siendo:

$I_{t}$ : índice de abundancia para el año $t$; $q$ : constante de proporcionalidad desconocida (en el caso en que el índice sea igual a la captura por unidad de esfuerzo (CPUE) esta constante es el denominado coeficiente de capturabilidad);

$\beta$ : potencia no negativa a la que debe elevarse la biomasa para obtener proporcionalidad con el índice;

$\eta_{t}$ : secuencia de variables aleatorias independientes, teniendo cada una distribución normal con media 0 y desvío estándar $\sigma_{\eta}$.

Y siendo además, $\bar{B}_{t}=w_{1} B_{t}+w_{2} B_{t+1}$ con $w_{1}$ $\geq 0$ y $w_{2} \geq 0$ conocidos y $w_{1}+w_{2}=1$.

Los casos más simples serían $\bar{B}_{t}=B_{t}\left(\operatorname{con} w_{1}\right.$ $\left.=1 \mathrm{y} w_{2}=0\right)$ o $\bar{B}_{t}=\left(B_{t}+B_{t+1}\right) / 2\left(\operatorname{con} w_{1}=w_{2}\right.$ $=1 / 2)$, estos dos casos se pueden ver en Punt y Hilborn (1996). El caso más general $\bar{B}_{t}=w_{1} B_{t}+$ $\left.w_{2}+B_{t+1}\right)$ sería adecuado cuando la información sobre el recurso, considerada al construir el índice de abundancia, corresponda principalmente a un intervalo de tiempo intermedio (conocido) dentro del año $t$.

Debe observarse que si se conocen los parámetros $\mu, \rho, \sigma_{\varepsilon}$ y los inobservables $B_{1}, P_{1}, \varepsilon_{2}, \varepsilon_{3} \ldots$, $\varepsilon_{n}$, entonces quedan unívocamente definidas las trayectorias de las biomasas $B_{t}$ y de las producciones excedentes $P_{t}$, para $1 \leq t \leq n$.

\section{Estimación bayesiana}

Supongamos que tenemos una serie de $n$ valores del índice de abundancia $I_{1}, I_{2}, \ldots, I_{n}$ y llamemos (la tilde indica el vector transpuesto):

$$
\begin{aligned}
& Y=\left(\ln \left(I_{1}\right),\left(\ln \left(I_{2}\right), \ldots,\left(\ln \left(I_{n}\right)\right)^{\prime} \mathrm{y}\right.\right. \\
& \theta=\left(\ln (q), \beta, \quad, \mu, \rho, \sigma_{\varepsilon}, P_{1}, B_{1}, \varepsilon_{2}, \varepsilon_{3} \ldots, \varepsilon_{n}\right)
\end{aligned}
$$

al vector de observaciones y al vector de parámetros, respectivamente. Tenemos que la verosimilitud de los datos, dado el vector de parámetros, está dada por:

$L(Y / \theta)=\frac{1}{(\sqrt{2 \pi})^{n}} \frac{1}{\sigma_{\eta}^{n}} e^{-\frac{1}{2} \sum_{t=1}^{n} \frac{\left(\ln \left(I_{t}\right)-\ln (q)-\ln \left(\bar{B}_{t}\right)\right)^{2}}{\sigma_{\eta}^{2}}}$

Según el trabajo de Walters y Ludwig (1994), podemos considerar a $q$ y $\sigma_{\eta}^{2}$ como nuisance parameters y eliminarlos de (7) mediante integración de $\ln (q)$ y $\sigma_{\eta}^{2}$, obteniendo:

$L\left(Y / \theta^{\prime}\right) \propto \mathrm{S}_{\mathrm{Z}}^{-(n-1)}$ 
siendo:

$\theta^{\prime}=\left(\beta, \mu, \rho, \sigma_{\varepsilon}, P_{1}, B_{1}, \varepsilon_{2}, \varepsilon_{3} \ldots, \varepsilon_{n}\right)^{\prime}$

$\mathrm{S}_{z}=\sqrt{\sum_{t=1}^{n}\left(z_{t}-\bar{z}\right)^{2} /(n-1)}$

$\operatorname{con} z_{t}=\ln \left(I_{t}\right)-\beta \ln \left(\bar{B}_{t}\right)$ у $\bar{z}=\frac{\sum_{t=1}^{n} z_{t}}{n}$.

Por su parte, suponiendo $\rho$ y $\sigma_{\varepsilon}$, independientes de $P_{1}$ y $B_{1}$, la prior del vector de parámetros $\theta$ ' la escribimos como:

$$
\begin{array}{r}
P\left(\theta^{\prime}\right)=P\left(\beta, \mu, \rho, \sigma_{\varepsilon}, P_{1}, B_{1}, \varepsilon_{2}, \varepsilon_{3} \ldots, \varepsilon_{n}\right) \\
=P(\beta) P(\mu) P(\rho) P\left(\sigma_{\varepsilon}\right) P\left(P_{1}, B_{1}\right) \\
P\left(\varepsilon_{2}, \varepsilon_{3} \ldots, \varepsilon_{n}\right) .
\end{array}
$$

Pero a su vez, teniendo en cuenta que estamos suponiendo que $\varepsilon_{2}, \varepsilon_{3} \ldots, \varepsilon_{n}$ son independientes y normalmente distribuidas, con media 0 y desvío estándar 1, tenemos que:

$P\left(\varepsilon_{2}, \varepsilon_{3}, \ldots, \varepsilon_{n}\right)=\frac{1}{(\sqrt{2 \pi})^{n-1}} e^{-\frac{1}{2} \sum_{t=2}^{n} \varepsilon_{t}^{2}}$.

Y de esta forma:

$$
\begin{gathered}
P\left(\theta^{\prime}\right)=P(\beta) P(\mu) P(\rho) P\left(\sigma_{\varepsilon}\right) P\left(P_{1}, B_{1}\right) \\
\frac{1}{(\sqrt{2 \pi})^{n-1}} e^{-\frac{1}{2} \sum_{t=2}^{n} \varepsilon_{t}^{2}} .
\end{gathered}
$$

Y entonces la distribución a posteriori del vector de parámetros, dados los datos, está dada por:

$$
\begin{array}{r}
P\left(\theta^{\prime} / Y\right) \propto \mathrm{S}_{\mathrm{z}}^{-(n-1)} P(\beta) P(\mu) P(\rho) P\left(\sigma_{\varepsilon}\right) P\left(P_{1}, B_{1}\right) \\
e^{-\frac{1}{2} \sum_{t=2}^{n} \varepsilon_{t}^{2}} \cdot(11)
\end{array}
$$

\section{Definición de las priors}

El conocimiento a priori que se pueda tener de los parámetros que conforman el vector de parámetros $\theta^{\prime}$, puede variar de una aplicación a otra de los modelos (1) y (2) con la ecuación de observación (5). Nosotros plantearemos el caso en donde el conocimiento disponible sea más bien difuso.

\section{Prior para $\beta$}

Como $\beta>0$, de acuerdo con la segunda regla de Jeffreys (Zellner 1987), se puede definir una prior poco informativa para $\beta$, considerando:

$$
\begin{aligned}
& \log (\beta) \sim \text { Uniforme }\left[\log \left(\beta_{1}\right), \log \left(\beta_{2}\right)\right], \\
& \quad \operatorname{con} \log \left(\beta_{1}\right) \leq \log (\beta) \leq \log \left(\beta_{2}\right) \text { y } \beta_{1}<\beta_{2} .
\end{aligned}
$$

\section{Prior para $\mu$}

Teniendo en cuenta que, en teoría, $\mu$ puede tomar valores tanto negativos como positivos, a partir de las reglas de Jeffreys definiremos la siguiente prior para $\mu$ :

$\mu \sim$ Uniforme $\left[\mu_{1}, \mu_{2}\right]$

$$
\operatorname{con} \mu_{1} \leq \mu \leq \mu_{2} \text { y } \mu_{1}<\mu_{2} \text {. }
$$

\section{Prior para $\rho$}

Teniendo en cuenta que satisface la desigualdad $-1<\rho<1$, entonces, a partir de la primera regla de Jeffreys (Zellner 1987) consideraremos como prior poco informativa para el coeficiente de autocorrelación serial $\rho$ a:

$$
\begin{aligned}
& \rho \sim \text { Uniforme }\left[\rho_{1}, \rho_{2}\right], \\
& \qquad \operatorname{con}-1<\rho_{1} \leq \rho \leq \rho_{2}<1 \text { y } \rho_{1}<\rho_{2} .
\end{aligned}
$$




\section{Prior para $\sigma_{\varepsilon}$}

Teniendo en cuenta (3) y (4), vemos que el coeficiente de variación de la producción excedente está dado por:

$C V=\frac{\sigma_{\varepsilon}}{\sqrt{\left(1-\rho^{2}\right)} \mu}$

de esta forma se tiene que:

$\sigma_{\varepsilon}=C V \sqrt{\left(1-\rho^{2}\right)} \mu$

teniendo en cuenta (13) y (14) y dado un valor promedio estimativo del coeficiente de variación $C V$, se puede definir:

$\sigma_{\varepsilon 1}=C V \sqrt{\left(1-\rho_{*}^{2}\right) \mu_{1}}$

$\sigma_{\varepsilon 2}=C V \sqrt{\left(1-\rho_{* 2}^{2}\right) \mu_{2}}$

siendo $\rho_{*_{1}}=\max \left\{\left|\rho_{1}\right|,\left|\rho_{2}\right|\right\}$ y $\rho_{*_{2}}=\min \left\{\left|\rho_{1}\right|,\left|\rho_{2}\right|\right\}$.

Por otra parte, siendo $\sigma_{\varepsilon}>0$ y teniendo en cuenta nuevamente la segunda regla de Jeffreys, se puede considerar una prior para $\sigma_{\varepsilon}$ de la forma:

$\log \left(\sigma_{\varepsilon}\right) \sim$ Uniforme $\left[\log \left(\sigma_{\varepsilon 1}\right), \log \left(\sigma_{\varepsilon 2}\right)\right]$,

con $\log \left(\sigma_{\varepsilon 1}\right) \leq \log \left(\sigma_{\varepsilon}\right) \leq \log \left(\sigma_{\varepsilon 2}\right)$ y $\sigma_{\varepsilon 1}<\sigma_{\varepsilon 2}$.

Prior para $P\left(P_{1}, B_{1}\right)$

Teniendo en cuenta que $P\left(P_{1}, B_{1}\right)=P\left(B_{1} / P_{1}\right)$ $P\left(P_{1}\right)$, se ve que para definir una prior para $\left(P_{1}\right.$, $\left.B_{1}\right)$, podemos definir las priors de $B_{1}$ condicionadas a estados dados de $P_{1}$ y una prior para $P_{1}$. Pero como estamos considerando el caso de definición de priors no informativas, podemos definir las priors de $B_{1}$ condicionadas a estados dados de $P_{1}$ sin tener en cuenta $P_{1}$ (observar que si estuviéramos en condiciones de definir priors de $B_{1}$ condicionadas a estados dados de $P_{1}$, esto implicaría poseer mucha información sobre la distribución conjunta de $\left(P_{1}, B_{1}\right)$ y estamos asumiendo que éste no es el caso). Por lo tanto consideraremos a $P_{1}$ y $B_{1}$ independientes. De esta forma $P\left(P_{1}, B_{1}\right)$
$=P\left(B_{1}\right) P\left(P_{1}\right)$ y por lo tanto para definir una prior no informativa para $\left(P_{1}, B_{1}\right)$, basta con definir priors poco informativas para $P_{1}, \mathrm{y} B_{1}$ en forma independiente. Finalmente, teniendo en cuenta las reglas de Jeffreys, definiremos las siguientes priors para $P_{1}$ y $B_{1}$ :

$P_{1} \sim$ Uniforme $\left[P_{11}, P_{12}\right]$,

$$
\text { con } P_{11} \leq P_{1} \leq P_{12} \text { y } P_{11}<P_{12}
$$

$\log \left(B_{1}\right) \sim$ Uniforme $\left[\log \left(B_{11}\right), \log \left(B_{12}\right)\right]$,

con $\log \left(B_{11}\right) \leq \log \left(B_{1}\right) \leq \log \left(B_{12}\right)$ y $B_{11}<B_{12}$.

\section{Algoritmo SIR}

Para el cálculo de la distribución a posteriori $P\left(\theta^{\prime} / Y\right)$, utilizaremos la función definida en (10) dado que esta es proporcional y proponemos el algoritmo SIR a los efectos de obtener una solución numérica. Consideramos el algoritmo SIR por su sencillez y fácil implementación y porque además su convergencia depende solo de la validez de la Ley de los Grandes Números (Bernardo y Smith 2000) y por lo tanto no depende de condiciones restrictivas o complejas difíciles de satisfacerse en la práctica.

Para aplicar el algoritmo SIR planteamos considerar como función de importancia a la prior $P\left(\theta^{\prime}\right)$ dada en (10), con las priors para cada parámetro definidas en (12), (13), (14), (19), (20) y (21). Teniendo en cuenta lo dicho, los pasos a seguir son los siguientes:

1) Seleccionar un número $m_{0}$, para definir el tamaño de la muestra de importancia y un número, $m<m_{0}$ para definir el tamaño de la muestra de remuestreo $\left(m_{0}\right.$ puede ser por ejemplo 1.000 .000 y $m$ 10.000).

2) Para $k=1$ hasta $m_{0}$ :

2.1. Sortear valores de los parámetros $\beta, \sigma_{\varepsilon}^{2}, \rho$, $P_{1}, B_{1}$, a partir de (10), (11), (18), (19) y 
(20), respectivamente y de los inobservables $\varepsilon_{2}, \varepsilon_{3} \ldots, \varepsilon_{n}$, independientes y cada uno con distribución normal con media $0 \mathrm{y}$ desvío estándar 1 . Al vector de parámetros obtenido llamarlo $\theta^{\prime}$.

2.2. Efectuar una prueba de admisibilidad biológica básica para el vector sorteado en el paso 2.1., verificando que: $B_{t}>C_{t}$ para $t=$ $0,1, \ldots, n$. Calculando las biomasa $B_{t}$ a partir de (1) y (2).

2.3. Calcular la tasa de importancia:

$$
w_{k}= \begin{cases}0 & \text { si } \theta^{\prime k} \text { no es admisible de } \\ \text { acuerdo con el test } & \text { efectuado en } 2.2 . \\ S_{z}^{-(r-1)} & \text { si } \theta^{\prime k} \text { es admisible de } \\ \text { acuerdo con el test } \\ \text { efectuado en } 2.2 .\end{cases}
$$

3) Sortear una muestra de tamaño $m$, de la muestra de tamaño $m_{0}$ original, con reemplazo y probabilidades proporcionales a $w_{k}$.

4) Calcular el coeficiente de variación del valor medio de las tasas de importancia $w_{1}, w_{2}, \ldots$, $w_{m_{0}}$ a partir de:

$c v(\bar{w})=\frac{1}{\sqrt{m_{0}}} \frac{S_{w}}{\bar{w}}$

siendo $\bar{w}$ y $S_{w}$, el valor medio y el desvío estándar de las tasas de importancia $w_{1}, w_{2}, \ldots$, $w_{m_{0}}$.

5) Si $c v(\bar{w})<0,04$ (McAllister y Kirchner 2002), se considera que el algoritmo SIR ha generado una muestra que honra la distribución a posteriori del vector de parámetros $\theta$ ' y finaliza el proceso de cálculo del algoritmo SIR. Si $c v(\bar{w})$ $\geq 0,04$ se vuelve al inicio de 2) y se rehacen los pasos 2), 3) y 4).

En caso de que en repetidos intentos no se pueda obtener convergencia, debe considerarse la posibilidad de incrementar el valor de $m_{0}$ en el paso 1) del algoritmo y rever minuciosamente la definición de las priors.

\section{Análisis de riesgo}

Se estima el riesgo de que se produzca disminución de biomasa el año siguiente $(n+2)$ al año de evaluación $(n+1)$, con evaluación de la probabilidad correspondiente a dicho riesgo, $\mathrm{P}(B(n+2)$ $<B(n+1))$. Para simplificar consideraremos que la evaluación la estamos efectuando el $1^{\circ}$ de enero del año en curso y las biomasas corresponden al $1^{\circ}$ de enero.

\section{Ejercicio de simulación}

Los datos utilizados corresponden a los estimados de captura desembarcada de corvina rubia (t) de la Argentina y Uruguay durante el período 2002 a 2010 (incluye capturas de la ZEE de ambos países) y el índice de abundancia denominado $\mathrm{AU}_{1}$, correspondiente a los valores de CPUE $\left(\mathrm{t} \mathrm{h}^{-1}\right)$ de 2002 a 2010, estimado a partir de los datos provenientes de la flota comercial argentina y uruguaya (Tabla 1).

A los efectos de evaluar la capacidad del MPECAS para representar y reproducir la dinámica poblacional en una situación conocida a priori se efectuó una simulación, considerando como modelo operacional un modelo de producción excedente de Schaefer representando la dinámica poblacional real (Hilborn y Walters 1992):

$B_{t+1}=B_{t}+r B_{t}\left(1-B_{t} / K\right)-C_{t}$

siendo:

$r$ : tasa intrínseca de crecimiento poblacional;

$K$ : capacidad de carga de la población.

Considerando además la relación entre el índice de abundancia y la biomasa, dada por:

$I_{t}=q B_{t} e^{\eta_{t}}$

con: 
Tabla 1. Serie de capturas desembarcadas de corvina rubia de la Argentina y Uruguay considerando información proveniente de aguas jurisdiccionales argentinas al norte de los $39^{\circ} \mathrm{S}$, aguas jurisdiccionales uruguayas, Río de la Plata y Zona Común de Pesca Argentino-Uruguaya y serie de índices de abundancia (CPUE estandarizada) correspondientes a datos de la Argentina y Uruguay $\left(\mathrm{AU}_{1}\right)$. Período 20022010.

Table 1. Series of white croaker landed catches from Argentina and Uruguay considering information derived from Argentine jurisdictional waters north of $39^{\circ} \mathrm{S}$, Uruguayan jurisdictional waters, de la Plata River and Argentine-Uruguayan Common Fishing Zone and series of abundance indices (standardized CPUE) corresponding to Argentine and Uruguayan $\left(A U_{1}\right)$. 2002-2010 period.

\begin{tabular}{lcc}
\hline Año & Captura $(\mathrm{t})$ & $\mathrm{AU}_{1}\left(\mathrm{~kg} \mathrm{~h}^{-1}\right)$ \\
\hline 2002 & 33.091 & 165,52 \\
2003 & 44.871 & 234,99 \\
2004 & 44.195 & 194,63 \\
2005 & 45.038 & 269,93 \\
2006 & 48.935 & 212,45 \\
2007 & 41.435 & 203,80 \\
2008 & 47.414 & 166,14 \\
2009 & 48.331 & 138,24 \\
2010 & 40.057 & 173,40 \\
\hline
\end{tabular}

$q$ : constante de proporcionalidad;

$\eta_{t}$ : error normal, con media 0 y desvío estándar $\sigma_{\eta}$.

En principio, se procedió a la estimación bayesiana, a partir del algoritmo SIR, de los parámetros del modelo de Schaefer. Los parámetros $q$ y $\sigma_{\eta}$ fueron tratados en principio como nuisance parameters y eliminados, por el procedimiento definido por Walter y Ludwig (1994), al efectuar la estimación bayesiana de los parámetros del modelo de Schaefer.

Las priors para el modelo de Schaefer fueron las siguientes: $\ln \left(B_{1}\right) \approx$ Uniforme $[\ln (100.000) ; \ln (300.000)]$

$\ln (r) \approx$ Uniforme $[\ln (0,20) ; \ln (0,40)]$

$\ln (K) \approx$ Uniforme $[\ln (400.000) ; \ln (800.000)]$.

Teniendo en cuenta las medias a posteriori (la media de la distribución a posteriori del parámetro) de los parámetros del modelo de Schaefer, se obtuvieron las siguientes estimaciones puntuales de los mismos:

$B_{1}=226.477$ (biomasa en 2002)

$K=589.615$

$r=0,3031$.

Y teniendo en cuenta el mejor ajuste mínimo cuadrático del índice teórico a los valores observados del índice de abundancia, se obtuvieron los valores:

$q=0,000883348$

$\sigma_{\eta}=0,1809$.

A partir de los parámetros (26) y (27) el modelo de Schaefer (23) fue considerado como modelo operacional en el ejercicio de simulación. De esta forma, teniendo en cuenta los valores de biomasa para los años 2002-2010, dadas por el modelo (23), se generaron valores del índice de abundancia para los años 2002-2010, a partir de la ecuación:

$I_{t}=q B_{t} e^{\varepsilon_{t}-\sigma_{\eta}^{2} / 2}$.

Las priors utilizadas se detallan a continuación:

$\ln \left(B_{1}\right) \approx$ Uniforme $[\ln (100.000) ; \ln (300.000)]$

$P_{1} \approx$ Uniforme[25.000; 55.000] 
$\mu \approx$ Uniforme[25.000; 55.000]

$\rho \approx$ Uniforme $[0,50 ; 0,80]$

$\ln \left(\sigma_{\varepsilon}\right) \approx$ Uniforme $[\ln (6.000) ; \ln (10.000)]$.

Para cada conjunto de valores del índice de abundancia se efectuó la estimación bayesiana (algoritmo SIR, $m_{0}=100.000, m=2.000$ ) de las distribuciones a posteriori de los parámetros $B_{t} \mathrm{y}$ $P_{t}$ del MPECAS, para $t=1,2, \ldots, 9$, cubriendo los años 2002, ..., 2010 y a partir de estas distribuciones se calcularon las medias a posteriori, para cada año $t$, para cada simulación $s, \bar{B}_{t}^{s}$ y $\bar{P}_{t}^{s}$. Los promedios de $\bar{B}_{t}^{s}$ y $\bar{P}_{t}^{s}$ sobre las 1.000 simulaciones, $\bar{B}_{t}$ y $\bar{P}_{t}$, fueron confrontados con los correspondientes parámetros del modelo operacional de Schaefer.

\section{Ejemplo de aplicación}

Los datos utilizados son los mismos que los considerados en el ejercicio de simulación y se encuentran consignados en la Tabla 1. En el ejemplo de aplicación se confrontaron las CMBA, para los riesgos del 5 y 10\% de disminución de biomasa el año siguiente al año de evaluación, correspondientes a la evaluación del recurso corvina rubia efectuada a partir del modelo de producción excedente de Schaefer, con los mismos valores correspondientes a la evaluación efectuada a partir del MPECAS. Para ambos modelos la estimación de parámetros se efectuó en un contexto bayesiano a partir del algoritmo SIR. Las priors para el modelo de Schaefer y el MPECAS fueron las mismas que las consignadas en (25) y (29), respectivamente.

\section{RESULTADOS Y DISCUSIÓN}

\section{Simulación}

En la Figura 1 se puede ver la trayectoria de biomasa del modelo de Schaefer (calculada a par-

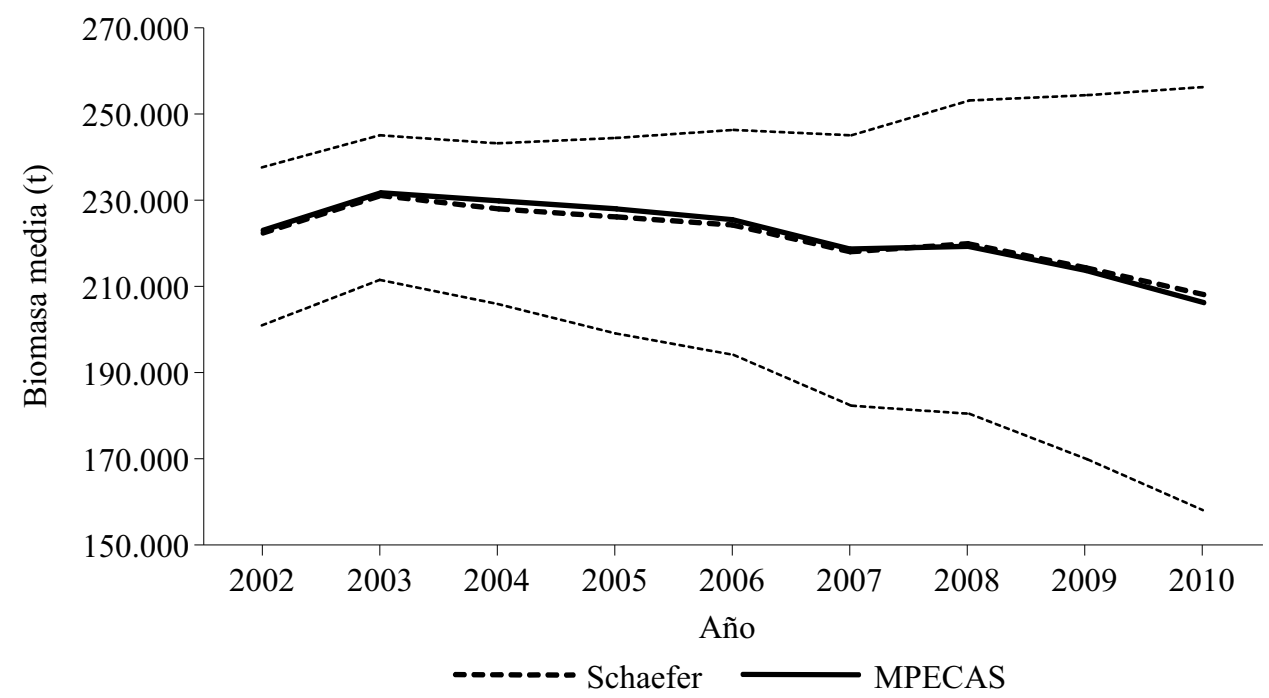

Figura 1. Trayectorias de biomasa del modelo operacional de Schaefer y del Modelo de Producción Excedente con Autocorrelación Serial (MPECAS). Las líneas de puntos indican los límites de confianza inferior y superior del 95\% derivados de la simulación.

Figure 1. Biomass trajectories of the Schaefer operational model and the Surplus Production Model with Serial Autocorrelation (MPECAS). The dotted lines indicate the 95\% lower and upper confidence limits derived from the simulation. 
tir de (23) con los parámetros (26)) y la trayectoria de biomasa media del MPECAS obtenida a partir de la simulación. Para cada año, el valor de biomasa media correspondiente al MPECAS es el promedio (sobre las 1.000 simulaciones) de las medias a posteriori para cada simulación, obtenidas éstas promediando los 2.000 valores de biomasa correspondientes a la etapa de remuestreo del algoritmo SIR. Como se observa las trayectorias difieren muy poco y la incertidumbre es menor para los primeros años debido al conocimiento a priori (si bien difuso) sobre el nivel de biomasa correspondiente a 2002. Debe observarse, no obstante, que la semiamplitud del intervalo de confianza para 2010 es solo un $26 \%$ de la biomasa media del MPECAS.

En la Figura 2 se puede ver la trayectoria de la producción excedente calculada a partir del modelo de Schaefer y la trayectoria de la producción excedente media del MPECAS obtenida a partir de la simulación. El procedimiento para el cálculo de ésta última es el mismo que el utilizado para calcular la trayectoria de biomasa media del modelo MPECAS.
La trayectoria de la producción excedente para el modelo de Schaefer es monótona decreciente, debido a que en el modelo de Schaefer la producción excedente es función de la biomasa y dependiendo de los niveles de biomasa, si decrece la biomasa también decrece la producción excedente. La trayectoria de la producción excedente media para el MPECAS se muestra más estable y en nivel de incertidumbre es más o menos constante, siendo la amplitud de los intervalos de confianza en promedio un $19 \%$ de la producción excedente media. La mayor diferencia entre el modelo de Schaefer y el MPECAS se da en 2010, pero el error porcentual es solo de un $12,55 \%$. Además, la trayectoria de las producciones excedentes del modelo de Schaefer está contenida en la banda de confianza asociada con la trayectoria de las producciones excedentes medias del MPECAS (Figura 2).

\section{Ejemplo de aplicación}

Las curvas de riesgo de disminución de biomasa de 2012 con respecto a 2011 se muestran en la Figura 3. En esta figura se han graficado conjun-

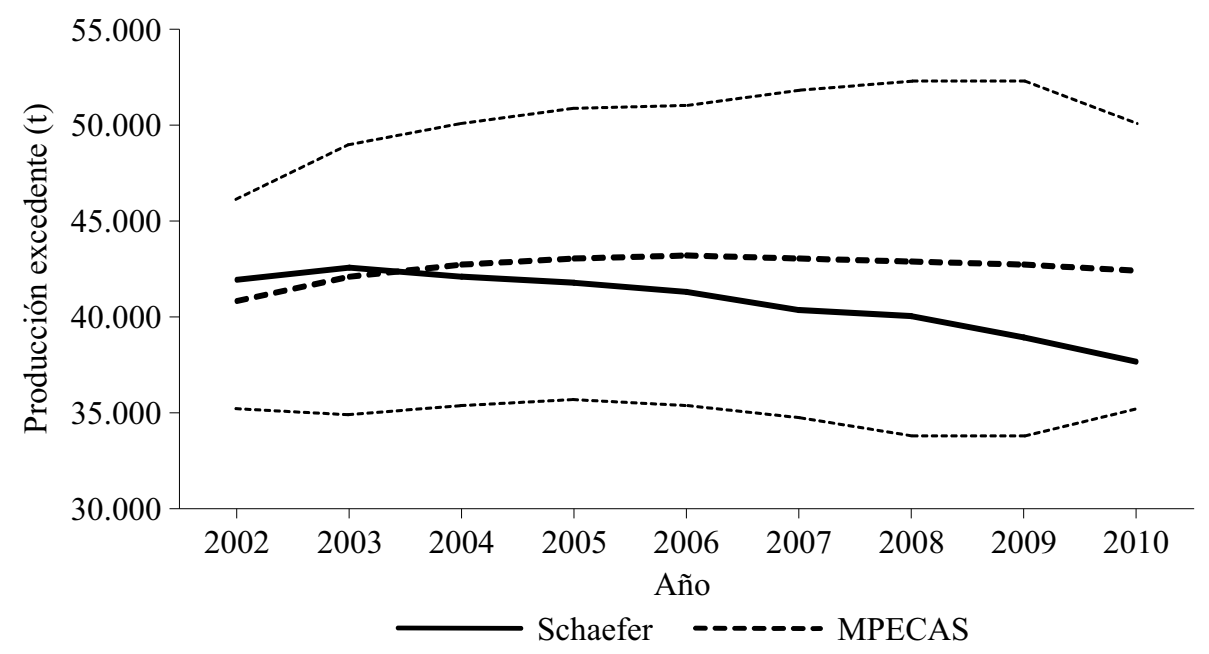

Figura 2. Trayectorias de la producción excedente del modelo operacional de Schaefer y del Modelo de Producción Excedente con Autocorrelación Serial (MPECAS) Las líneas de puntos indican los límites de confianza inferior y superior del 95\% derivados de la simulación.

Figure 2. Surplus production trajectories of the Schaefer operational model and the Surplus Production Model with Serial Autocorrelation (MPECAS). The dotted lines indicate the 95\% lower and upper confidence limits derived from the simulation. 


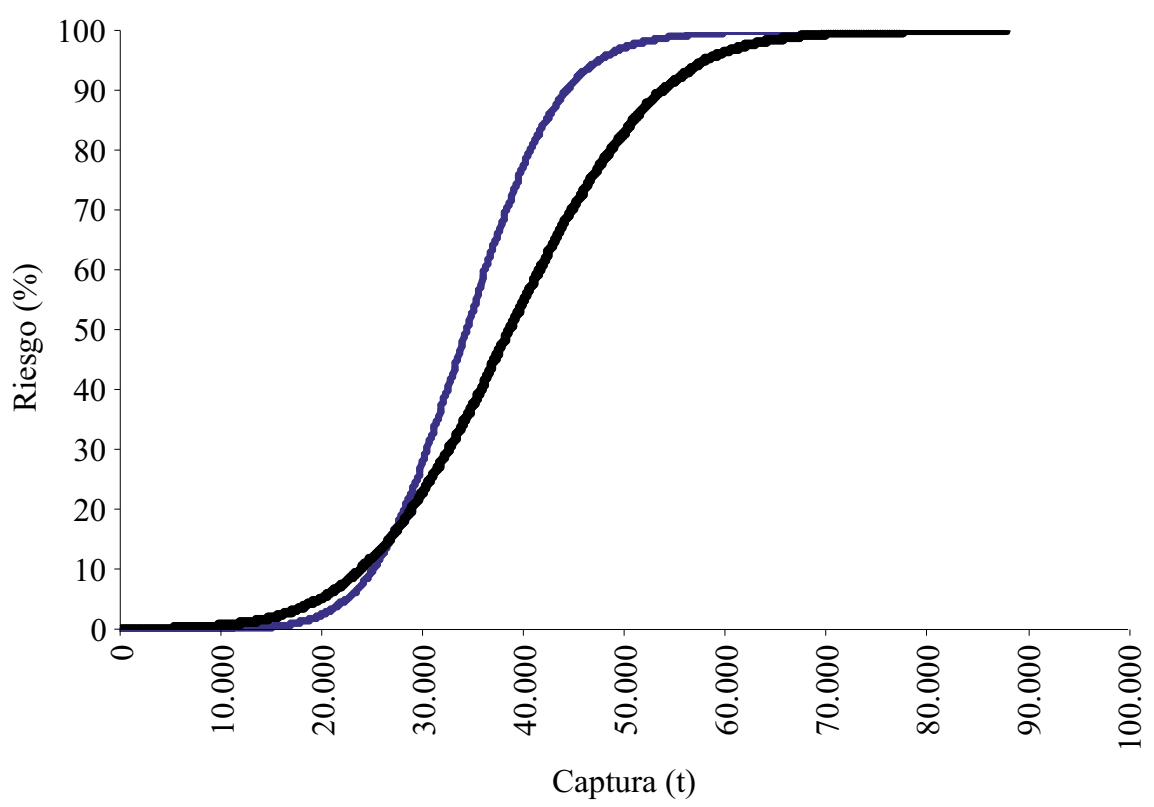

Schaefer

MPECAS

Figura 3. Curvas de riesgo $\left(\mathrm{B}_{2012}<\mathrm{B}_{2011}\right)$ obtenidas con el Modelo de Dinámica de Biomasa de Schaefer y el Modelo de Producción Excedente con Autocorrelación Serial (MPECAS) considerando el índice $\mathrm{AU}_{1}$ en el período $2002-2010$.

Figure 3. Risk Curves $\left(\mathrm{B}_{2012}<\mathrm{B}_{2011}\right)$ obtained with the Schaefer Biomass Dynamics Model and the Surplus Production Model with Serial Autocorrelation (MPECAS) considering the $A U_{1}$ index in the 2002-2010 period.

tamente la curva de riesgo del modelo de producción excedente de Schaefer y la obtenida a partir del MPECAS.

Cuando se compara la curva de riesgo $\left(B_{2012}<\right.$ $\left.B_{2011}\right)$ obtenida a partir del modelo de Schaefer con la obtenida a partir del MPECAS (calculadas con el índice de abundancia $\mathrm{AU}_{1}$ en el período 2002-2010), se aprecia que la curva correspondiente al MPECAS determina riesgos mayores que la curva correspondiente al modelo de Schaefer al considerar riesgos entre 0 y $10 \%$, comportándose por lo tanto el MPECAS más conservador y precautorio que el modelo de Schaefer en éste rango de riesgos, generalmente considerados en las aplicaciones. Para riesgos mayores se invierte el comportamiento de ambos modelos.

En la Tabla 2 se muestran las CMBA para los riesgos del 5 y $10 \%$ considerando el riesgo de disminución de biomasa de 2012 con respecto a 2011, obtenidas a partir del modelo de Schaefer y
Tabla 2. Capturas precautorias al 5 y $10 \%$ de riesgo $\left(\mathrm{B}_{2012}<\right.$ $\mathrm{B}_{2011}$ ) estimadas con el Modelo de Producción Excedente de Schaefer y el Modelo de Producción Excedente con Autocorrelación Serial (MPECAS) considerando la serie de abundancia $\mathrm{AU}_{1}$ en el período 2002-2010.

Table 2. Precautionary catches at 5 and $10 \%$ risk $\left(B_{2012}<\right.$ $\left.B_{2011}\right)$ estimated with the Schaefer Surplus Production Model and the Surplus Production Model with Serial Autocorrelation (MPECAS) considering the $A U_{1}$ series in the 2002-2010 period.

\begin{tabular}{lcc} 
Modelo & Captura al 5\% & Captura al 10\% \\
\hline Schaefer & 22.500 & 25.200 \\
MPECAS & 19.700 & 23.800 \\
\hline
\end{tabular}

el MPECAS. Si bien, como cabría esperar, los valores de las capturas precautorias presentan algunas diferencias, al comparar el MPECAS con 
el modelo de Schaefer y tomando a este último como referencia, éstas son menores al $13 \%$ en el caso de las capturas al nivel de riesgo del 5\% y menores al 6\% en el caso de las capturas al nivel de riesgo del 10\%. El MPECAS, como se dijo, se muestra moderadamente más conservador para estos niveles de riesgo considerados, presentando capturas menores que el modelo de Schaefer.

\section{CONCLUSIONES}

El MPECAS es un modelo simple que no hace ningún supuesto restrictivo sobre la relación entre la producción excedente y la biomasa del recurso como sí lo hacen, por ejemplo, los modelos de Schaefer, Fox y Pella y Tomlinson (Hilborn y Walters 1992). El mismo considera como único supuesto que la producción excedente presenta correlación serial. De esta forma, se plantea que la producción excedente de cada año tiene correlación con las producciones excedentes de años previos, disminuyendo el grado de correlación a medida que nos alejamos en el tiempo del año en consideración.

El mismo requiere para su aplicación a la evaluación de un recurso y la estimación del vector de parámetros, el conocimiento de un índice de abundancia proporcional a una potencia de la abundancia media real del recurso, pudiendo efectuarse fácilmente la estimación de parámetros en un contexto bayesiano.

A partir del MPECAS se pueden efectuar proyecciones de biomasa en un corto-mediano plazo (de 1 a 5 años) y evaluar situaciones futuras probables del recurso ante una secuencia de capturas preestablecida y fijando los niveles de riesgo admisibles determinar las CMBA.

El MPECAS se destaca porque en su definición respeta la estructura básica de un modelo de producción excedente, pero carece de supuestos fuertes y restrictivos, los cuales no siempre son fácilmente justificables.

\section{AGRADECIMIENTOS}

Agradecemos a la Dra. Claudia R. Carozza, Jefa del Programa de Pesquerías de Peces Demersales Costeros, la cual gentilmente brindó los datos que permitieron desarrollar el ejemplo de aplicación. Contribución INIDEP N 2161.

\section{BIBLIOGRAFÍA}

Bernardo JM, Smith AFM. 2000. Bayesian Theory. Chichester: Wiley \& Sons. 586 p.

Harvey AC. 1981. Time Series Models. Oxford: Philip Allan Publishers Limited. 229 p.

Hilborn R, Walters CJ. 1992. Quantitative Fisheries Stock Assessment. Choice, Dynamics and Uncertainty. Nueva York: Chapman \& Hall. 570 p.

McAllister M, Kirchner C. 2002. Accounting for structural uncertainty to facilitate precautionary fishery management: illustration with namibian orange roughy. Bull Mar Sci. 70 (2): 499-540.

Punt E, Hilborn R. 1996. BIODYN. Biomass dynamic models. User's manual. FAO Comput Inf Ser (Fish). 62 p.

Walters C, Ludwig D. 1994. Calculation of Bayes Posterior Probability Distributions for Key Population Parameters. Can J Fish Aquat Sci. 51: 713-722.

ZELLNER A. 1987. An Introduction to Bayesian Inference in Econometrics. Malabar: Robert E. Krieger Publishing Company. 431 p.

Recibido: 30-10-2017

Aceptado: 20-02-2019 
